\title{
Study on Automatic Measuring Method of Total Sugar Content in Chlortetracycline Fermentation Tank
}

\author{
XiaoYu Qu ${ }^{1, \mathrm{a}}$, Li Wang ${ }^{1, \mathrm{~b}}$, Lei Wu ${ }^{1, \mathrm{c}}$,Xiangguang Chen ${ }^{1, \mathrm{~d}, *}$, Minpu Yao ${ }^{2, \mathrm{e}}$, Qing \\ $\mathrm{Yu}^{2, f}$, Suyi Huang ${ }^{2, \mathrm{~g}}$, Biao Zhou ${ }^{2, \mathrm{~h}}$
}

${ }^{1}$ School of Chemical Engineering and Environment,Beijing Institute of Technology,Beijing ,100081, China

${ }^{2}$ Pucheng Zhengda Fujian Biochemical Co. Ltd., Fujian, Pucheng, 353400, China

a aprilxiaoyu@126.com, ${ }^{b}$ wangli_hb@qq.com, ${ }^{c} 570068938 @ q q . c o m,{ }^{\mathrm{d}^{*}}$ Corresponding, Author, xgc1@bit.edu.cn, ${ }^{\mathrm{e}}$ yaominpu@yahoo.cn, fsdyq@ct-bio.com, ${ }^{\mathrm{g}} \mathrm{hsy} 0531 @ 163 . c o m,{ }^{\mathrm{h}}$ zhoub@ct-bio.c om

Keywords: CTC fermentation tank, Measurement of total sugar content, Refractive method, Microwave method, TMS320F2812.

\begin{abstract}
In order to realize the automatic measurement of total sugar content in the process of chlortetracycline (CTC) fermentation and optimize the carbohydrate supplement adjustment process, we designed the digital signal processor TMS320F2812 as the core of automatic measurement controller. The functions of automatic sampling, filtering and communication between the controller and the host computer are implemented. Moreover, refraction and microwave were used respectively to measure the total sugar content of fermented liquid. The automatic measuring system software was programmed based on language C in CCS (code composer studio). Serial port communication between DSP and PC provides for convenient query and data display. The experimental results show that the refraction method can meet the needs of the automatic measurement on the total sugar content in the process of CTC fermentation.
\end{abstract}

\section{Introduction}

Total sugar content refers to reducing glucose, fructose, pentose sugar, lactose, the sugar that can be hydrolyzed to reducing the monosaccharide under the special condition, maltose, and partially hydrolyzed starch[1]. Fermentation engineering is an important part of modern industrial production, due to the content of many kinds of chlortetracycline fermented liquid and high viscosity. The measurement of total sugar content in CTC fermentation tank is so far still based on artificial sampling analysis with huge labours and long analysis process. Therefore, the realization of automatic measuring of total sugar content for CTC fermentation is of important practical significance.The measuring method for total sugar content in fermentation tank can be generally divided into two major categories, namely:

i) Chemical test: Qingyu Lin and other scholars made use of Fehling method to test the reducing sugar in horseshoes[2]. But the results are greatly influenced by equipment instruments and operators. Ping $\mathrm{Wu}$ and other co-workers used iodine volume method to test the total sugar content in the wine[3]. However, the measurement of total sugar content in the actual application result is affected by temperature seriously and the process is complicated. Qiuju Wang used anthrone-sulfuric acid method for measuring the polysaccharide content in the yeast[4]. But it is only suitable for little liquid measurement.

ii) Industrial test: Wenhua Miao scholar tested total sugar content with ultrasonic wave method[5]. Nevertheless, a large amount of data in the process of experiment is needed as the foundation of the fitted curve. And the inevitably error in sound velocity measurement will affect the final results. Hongyan Guo and other scholars utilized refraction method, DNS method and Fehling method to test three consecutive batches of abamectin sugar content in the fermented liquid respectively[6].The relevant operation process in the DNS method is complex. Human configuring reaction reagent is demanded in Fehling method. Moreover, the measuring data is not accurate. 
Nevertheless, some researchers prove that microwave can be used to measure the concentration of various substances through experiments. For example, Makoto, Atsushi and Kiichiro use it to test the sugar content in apples[7]. Ulrich Klute takes advantage of it in the automatic system of boiled sugar crystallization cans[8].

In this paper, the automatic measurement system is based on TMS320F2812, which is designed for automatic sampling and filtering for the liquid of CTC fermentation. And then with the use of microwave and refractive methods, we implement the automatic measuring of total sugar in the fermented liquid and realize the communication between the controller and PC.

\section{Design for automatic measuring system}

As the core of the automatic measuring system controller, TMS320F2812 is used to realize automatic switch control of the various electromagnetic valves, centrifugal pump and fan. And the serial communication with the host computer is used to control the whole process. Fig. 1 is the diagram for total sugar automatic measuring system.

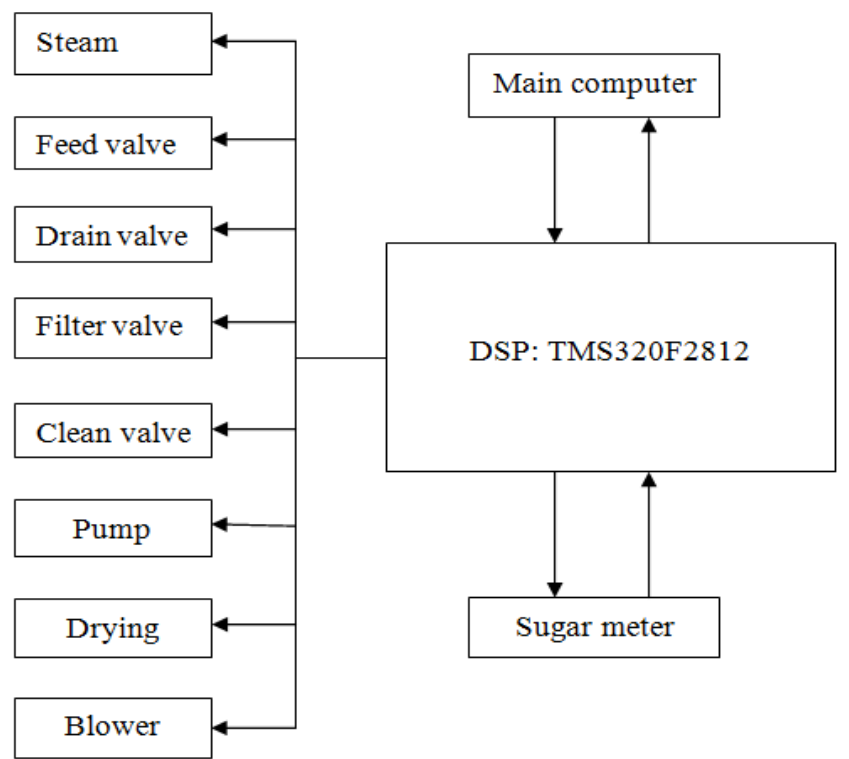

Figure.1. The diagram for total sugar measuring system in CTC fermentation tank

The power supply of the electromagnetic valve is $24 \mathrm{~V}$ and which of the fan centrifugal pump is $220 \mathrm{~V}$. While the voltage of the GPIO output port of TMS320F2812 is only $3.3 \mathrm{~V}$. Therefore, the isolation protection circuit is of great importance. TLP521 and ULN2003 are utilized to realize electrical isolation protection and driving function. Fig.2 is hardware circuit diagram for port control.

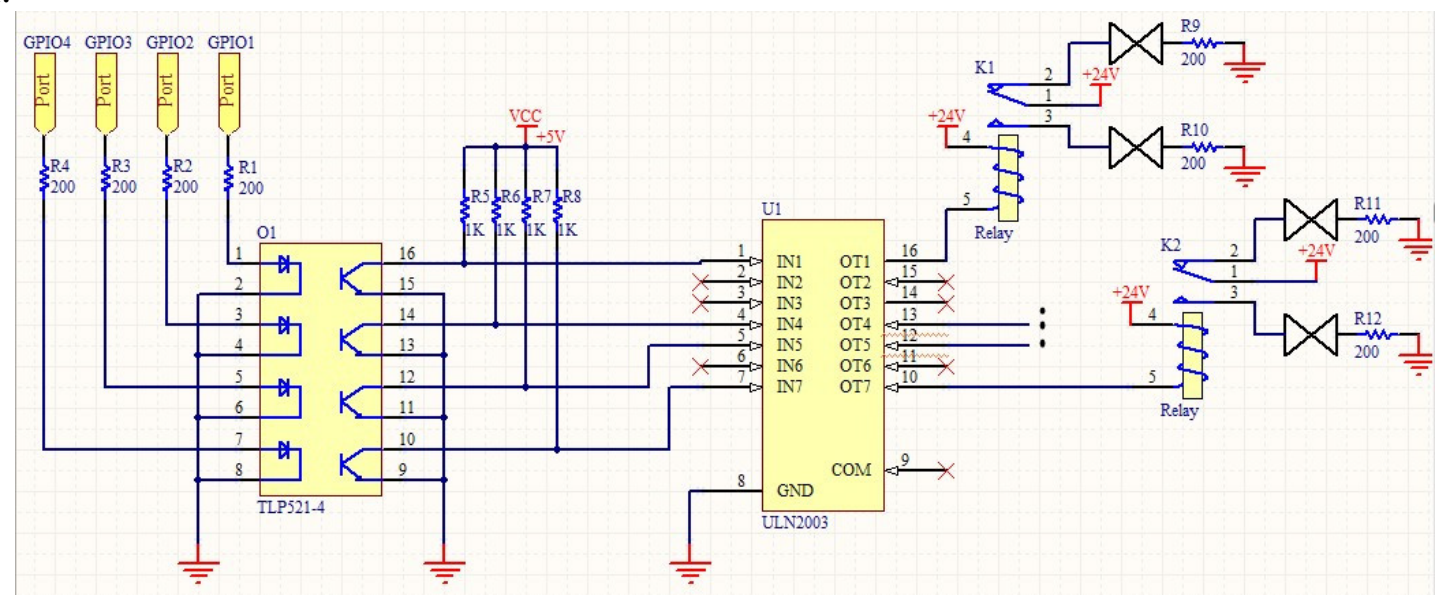

Figure.2. Control circuit in output port of TMS320F2812 


\section{Measuring principle of total sugar content in the fermentation tank}

The measuring principle of microwave method is as following. Microwave transmission in the measured liquid passed through water molecules. Spin polarization will appear in water molecules in the microwave field, which consumes microwave energy[9].As Eq.1 shows, usually dielectric constant $\varepsilon$ 'represents the performance storing potential energy of the material. $\varepsilon$ "stands for the consumption of the material. $\varepsilon^{*}$ is the complex permittivity.

$$
\varepsilon^{*}=\varepsilon^{\prime}-\mathrm{j} \varepsilon^{\prime \prime} \text {. }
$$

Microwave measurement is based on the size of the complex dielectric constant to determine the samples' content [10]. According to the theory of electromagnetic wave propagation, when microwave passes through a length $(\mathrm{L})$ of specimen, the amplitude attenuation $(\mathrm{E})$ is

$$
E=8.686 \frac{L \pi}{\lambda} \sqrt{\frac{\varepsilon^{\prime}-P}{2}\left[\sqrt{1+\left(\frac{\varepsilon^{\prime \prime}}{\varepsilon^{\prime}-P}\right)^{2}}-1\right]} .
$$

Where, ${ }^{P}=\left(\lambda / \lambda_{c}\right)^{2}, \lambda$ is the wavelength in the free space, referred to the operating wavelength. As Eq.2 shows, the complex dielectric constant value is different with samples of different sugar content.

Abel refraction method is mainly founded on the refraction phenomenon of light. Refractive index of the solution is closely linked to the wavelength of incident light and the temperature. It can be shown in Eq.3.

$$
n=f(\lambda, T, C) .
$$

Where, $\lambda$ is the wavelength of incident light, $\mathrm{T}$ is the temperature, $\mathrm{C}$ stands for the sugar density of the liquid. When the temperature $\mathrm{T}$ and the wavelength of the incident light $\lambda$ are determined, the corresponding relation between concentration $\mathrm{C}$ and refractive index $\mathrm{n}$ is

$$
C=f^{-1}(n)
$$

As is shown in Eq.4, by measuring the refractive index, we can indirectly calculate the consistence of sugar solution.

\section{Program design of testing system for total sugar content}

The controller of automatic measuring system for total sugar content in CTC fermentation liquor is mainly working on valve, fan and centrifugal pump switch operations and the switch state. A series of control actions are accomplished by GPIO ports of the DSP. Program flow chart in the process of measuring total sugar content is as shown in Fig.3. The host system waits for the measuring command sent from DSP via a serial port. And then it carries on the follow-up work. 


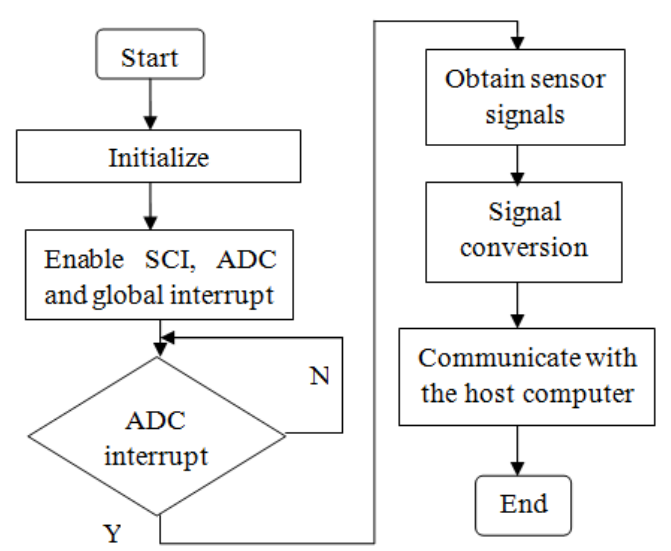

Figure.3. Program flow chart for testing total sugar content

Serial port communication mode between DSP and PC is conducted by SCI interruption of TMS320F2812. CPU interface of SCI is shown in Fig.4.

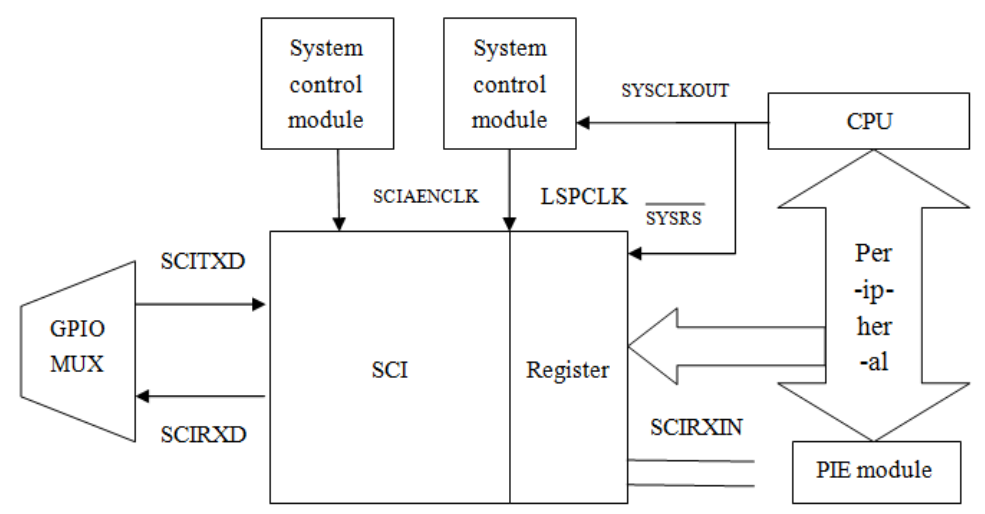

Figure.4. Block diagram of SCI CPU interface

SCITXDA and SCIRXDA realize the functions of data sending and receiving respectively that are correspond to the fourth bit and fifth bit of GPIOF module. The system clock of CPU is SYSCLKOUT. After low speed demarcation, its output will be low speed clock LSPCLK to supply SCI. SCI can produce two interrupts, SCIRXINTA and SCITXINTA, namely sending interrupt and receiving interrupt.

Serial communication is RS232 communication mode. Baud rate is 19200. A frame of data format sent between 2812 and the upper machine is as shown in Fig.5. LSB is the lowest bit, while MSB is the highest bit.

\begin{tabular}{|c|c|c|c|c|c|c|c|c|c|c|}
\hline $\begin{array}{c}\text { Start } \\
\text { bit }\end{array}$ & LSB & 2 & 3 & 4 & 5 & 6 & 7 & MSB & $\begin{array}{c}\text { Check } \\
\text { digit }\end{array}$ & $\begin{array}{c}\text { End } \\
\text { bit }\end{array}$ \\
\hline
\end{tabular}

Figure.5. A frame of data format for SCI

\section{Analysis of the experiment results}

As the sugar content to be tested in this project is less than $15 \%$, so the deploying sugar content of the experiment is controlled below 15\%. With microwave method, we test different content of sugar solution, and fit the data with MATLAB. As can be seen directly from Fig.6, the sugar content and the voltage collected have a linear relation. 


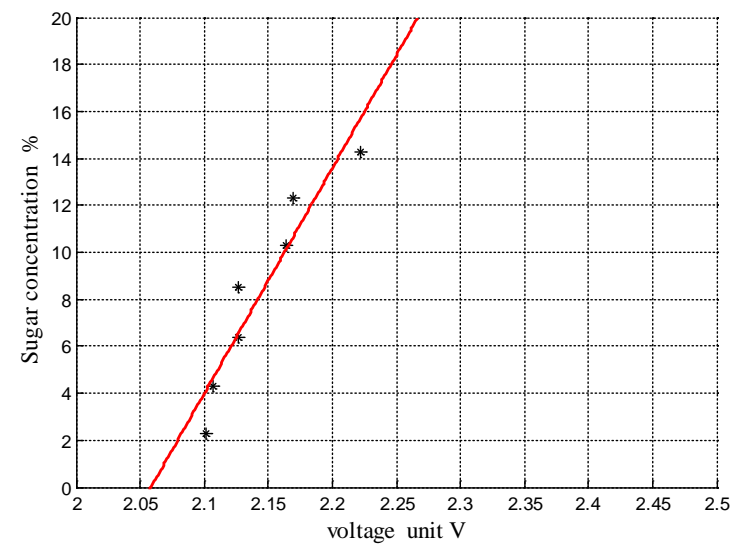

Figure.6. Fitting curve of testing data with microwave

Table.1 Microwave test the fitting values and errors

\begin{tabular}{cccccccc}
\hline concentration\% & 2 & 4 & 6 & 8 & 10 & 12 & 14 \\
\hline voltage $(\mathrm{V})$ & 2.101 & 2.107 & 2.127 & 2.127 & 2.164 & 2.170 & 2.22152 \\
fitting value & 3.357 & 4.019 & 6.118 & 6.074 & 10.073 & 10.643 & 16.1656 \\
error & 1.357 & 0.019 & 0.118 & -1.926 & 0.073 & -1.357 & 2.16561 \\
\hline
\end{tabular}

It is obvious in Table.1 that errors of microwave method are not very stable. But in the range of allowable error, some measuring data is very close to the real value of the total sugar content.

With refraction method, measuring data and the results are as shown in Fig.7. Where, the imaginary line with circle is the standard curve, solid line belt * is the measuring curve.

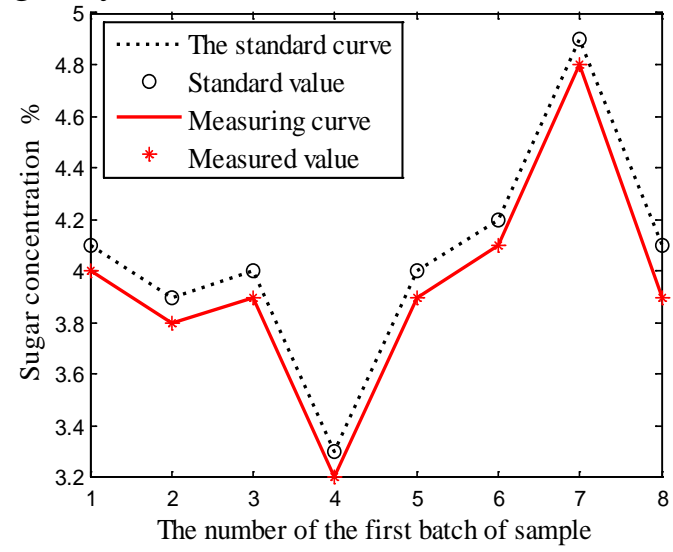

(a)the first batch

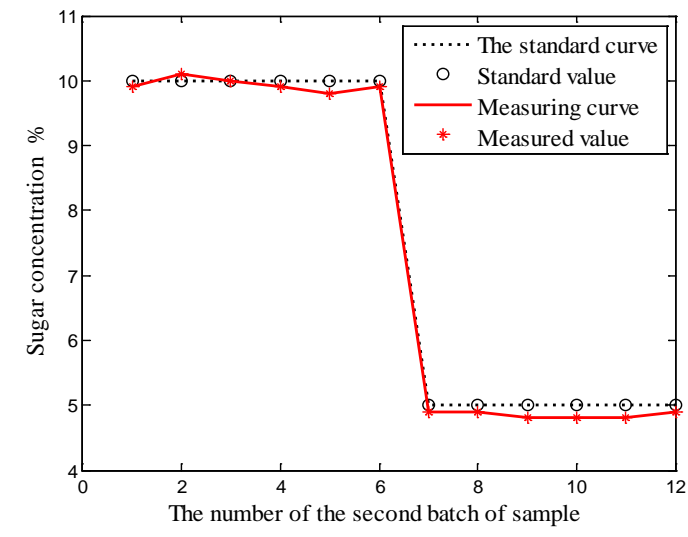

(b)the second batch

Figure.7. refraction method to measure total sugar results

\section{Conclusions}

On the basis of TMS320F2812, automatic measureing system of total sugar content in fermentation tank was designed. And both microwave and refraction methods were adopted to realize the measurement of total sugar content of fermented liquid. Through a serial port communication, data was displayed on the host computer. The experimental process was running well and the entire unit can meet the demand of real industries. In the process of testing total sugar content, the results based on refraction method are of higher precision than microwave method, with no electromagnetic interference from the outside world. Moreover, the feasibility and measurement accuracy can meet the requirements of real production process. 


\section{Acknowledgements}

The research work was supported by Pucheng Zhengda Fujian Biochemical Co. Ltd., Fujian, Pucheng 353400, China.

\section{References}

[1] Wu Xiaoyuan. Research on total sugar measurement of the liquid sugar in cane sugar processing[D]. South China University of Technology. 2013.

[2] Lin Qingyu,Zhang Zhi,Wei Xiaoping,etc.Automatic potentiometric titration method for the determination of total reducing sugar in the horseshoe[J]. Anhui Agricultural Sciences.2011, 39 (26), 16450-16451.

[3] Wu Ping, Zhao Wenjing, Xu Xiaojiao, etc. Discussions on detection methods of total sugar in wine[J]. China Brewing. 2011,226(1),163-165.

[4] Wang Qiuju, Xu Li, Cui Yizhe, etc. Anthrone - sulfuric acid method for determining the content of yeast polysaccharide[J]. China Animal Husbandry and Veterinary. 2007,34(1), 39-40.

[5] Miao Wenhua, Xu Xiaoyun, Zhou Bin, et al. Improvement of sugar production from potato pulp with microwave radiation and ultrasonic wave pretreatments[J]. Food Process Engineering.2014,37 (1),86-90.

[6]Guo Hongyan,Chen Haiyan. Three sugar content determination methods comparisons in Abamectin fermentation process[J]. Petrochemical Applications.2012,31(6),52-54.

[7] Makoto,Atsushi,Kiichiro.Non-destructive Measurement of Sugar Content in Apples using MillimeterWave Reflectometry[J].Infrared Milli Terahz Waves.2012, 33, 228-236.

[8] Ulrich Klute.Microwave measuring technology for the sugar industry[J]. International Sugar Journal.2007, 109, 1308-1309.

[9] G.Guarin,M.Hofmann,R.Weigel,et al. Determination of Sugar Concentration in Aqueous Solutions Using Ultra-Wideband Microwave Impedance Spectroscopy. Erlangen: IEEE, conference location: Seattle, WA. 2013, 1-4.

[10] Wenchuan Guo, Xinhua Zhu, Yi Liu, et al. Sugar and water contents of honey with dielectric property sensing[J]. Food Engineering.2010, 97, 275-281. 